\title{
A Proposed Approach to Classification and Novel Class Detection in Data Streams
}

\author{
Ms. Rimjhim Singh ${ }^{1}$, Manoj. B. Chandak ${ }^{2}$ \\ M.Tech Scholar, CSE Department, SRCOEM, Nagpur, India ${ }^{1}$ \\ Professor and Head, CSE Department, Nagpur, India ${ }^{2}$
}

\begin{abstract}
In the field of data mining, data streams play an important role. In order to make intelligent use of data streams, first we need to handle them properly. The notation data stream itself represents the nature of such data. They possess the property of being dynamic and continuous in nature. That is the data keeps on changing the features and properties with time. Due to the above mentioned properties of data streams, various challenges are posed by them to researchers. These challenges are infinite-length, concept-evolution, feature-evolution and concept-drift. Infinite-length is due to the continuous nature of data. Concept-evolution is due to the new emerging classes. Concept-drift is due to the drifting concept of the stream and feature-evolution is there because of the changing features. These challenges have been well studied by various researchers and they have proposed various approaches to handle them. In this paper we also try to propose a strategy based on strings to handle the problems of infinite-length, concept-evolution and concept-drift.
\end{abstract}

Keywords: mining, novel, concept-drift, concept-evolution

\section{INTRODUCTION}

Advances in the field of data storage have enabled the data into chunks and then train the model with the researches to store and handle huge amount of data in real recent data chunks available. As concept-drift occurs due time. This leads to data that may grow beyond limit and to the changes in the concept of stream with time, we need such data is usually referred to as stream data. While to detect these changes and update the model regularly talking about mining data, we mean handling streams of with them. Different researchers have proposed techniques data. Like classification is one of the problems that need to to update the model. Many of the proposed models assume be solved in data mining. Because of the continuous and that the number of classes present in the stream are fixed dynamic nature of data it becomes impossible to handle and continue with the classification procedure. But this is data streams with the strategies developed for handling not the case with data streams. As we know that conceptstatic data. Due to the above mentioned properties, data evolution is due to the occurrence of new classes in the streams pose different challenges to researchers. One of stream that are unknown to our system also. Hence such the major issues is infinite-length. Stream data may grow models will not be able to classify the instances belonging beyond limit and it is not feasible to store such huge to new classes that will lead to inefficient classification. So amount of historical data. Hence, it becomes impractical to the model needs to be trained on the recently occurring use this data for training our model. Stream data also new classes in the stream. As an example, in case of experiences changes in the underlying concept. This intrusion detection we need to train our model on new occurs when features of various classes present in the data class of attacks so that they are detected and classified at a start drifting towards new features slowly and the classes later stage. Because of this, traditional classifiers don't undergo the conceptual change. Third challenge is posed work efficiently with data streams, as they are not able to when the stream starts facing new concepts. By new mechanically detect the novel classes. Feature-evolution is concepts we mean, new classes that are unknown to our also handled by some researchers. We can handle it by system and stream start appearing in it at some later stage. keeping track of the new features and their count occurring For example, in case of intrusion detection, if we consider in the stream. We must also keep track of the old features each type of attack as a class label. When altogether new that are fading away from the stream. Here we need to type of attack will occur then it will be a new class label modify the feature vector of the model.

and we can say that concept-evolution is present. The In this paper we develop a strategy to handle data streams. instances belonging to these classes will not be classified We first form an ensemble of model. Secondly, try to by our system. Last one is feature-evolution, where new detect the outliers from the model. Third we try to separate features start appearing in stream and older features start out the outliers due to concept drift and handle them. fading away. We need to handle all these challenges in Lastly, we try to get the outliers occurring due to conceptorder to make efficient use of data streams.

Various strategies have been proposed by researchers to handle these problems. Most of these strategies are not able to handle the issues efficiently. In order to handle infinite-length problem various incremental learning techniques have been proposed. These techniques divide evolution and try to handle them.

\section{RELATED WORK}

Researchers have done a lot of work and given many strategies that can handle infinite-length and concept-drift effectively. But many of them fail to handle concept- 
evolution and feature-evolution efficiently. Various novel class detection are unable to detect the presence of incremental approaches have been proposed in this multiple novel classes simultaneously. But our technique direction. These approaches are basically of two types, can detect and handle multiple novel classes occurring namely single model incremental approach and hybridbatch incremental approach. In single model incremental approach ( proposed in [1]) use the concept of a single model. This model is continuously updated with the new features or data appearing in the stream data. The modification is done dynamically and regularly. But the main drawback of this approach is that the model becomes too vague and large. Its feature set expands up to a great extent and it loses its significance. Other approach, hybridbatch incremental approach (proposed in [2], [3]), uses a collection of models. In this technique each model is generated by using batch learning process. Recent data chunk is used to generate a model and then the models are updated dynamically. This approach is more efficient as it is easy to update the individual models. The models that become obsolete at some stage are discarded and are replaced by the newer models. While handling data streams we must know that outliers occur due to three main reasons, namely, concept-evolution, concept-drift and noise. We not only need to detect these outliers but we also need to find the cause of their occurrence. If the cause is known, only then we can develop strategy to handle the outliers. Otherwise it might happen we classify an instance as an outlier occurring due to concept-evolution while it originally occurred due to concept-drift and vice versa. In this way misclassification might occur. And if this will happen then the false alarm rate of the classifier will be high.

Spinosa et al (explained in [4]) proposed an approach that is able to handle concept-drift, infinite-length and conceptevolution also. It detected the novel classes by making use of clustering techniques. They created a model by using the clustering and this model was encompassed by the hyper-sphere. If a cluster was obtained, if it lied outside the hyper-sphere and if it also contained considerable number of instances then it was said to be a novel class. This technique is a one class classifier that is it assumes only one class as normal class. Rest all other classes are considered as novel. Hence it had a drawback that it can't be used with data having multiple classes. It also assumed that the shape of normal class instances in the feature space is convex. But this is not the case in real time situations.

simultaneously in the data

\section{PROPOSED APPROACH}

In the proposed approach we try to develop a technique that is based on strings to work with stream data. We try develop a classification model that is an ensemble of several models. Each sub-model will also work as a classification model.

While handling data streams we must know that in data streams we encounter two types of classes, namely, existing classes and novel classes. Let us assume that ' $L$ ' is an ensemble of classification models $\{\mathrm{M} 1, \mathrm{M} 2, \mathrm{M} 3, \ldots$. , Mn\} that we have developed. Following are some definitions that are frequently used in our approach.

Definition 1: Existing Class: Assume there is a class named ' $C$ '. ' $C$ ' is said to be an existing class if at least one of the models ' $\mathrm{Mi}$ ' belonging to ensemble of models ' $\mathrm{L}$ ' has been trained on class ' $\mathrm{C}$ '. Otherwise it is not an existing class.

Definition 2: Novel Class: Assume there is a class named ' $\mathrm{N}$ ' in our data. If none of the models belonging to the ensemble of models ' $L$ ' is trained on ' $L$ ', then ' $N$ ' is said to be a novel class. In short a novel class is completely unknown to our system.

Definition 3: Outliers: Assume there is an instance named ' $\mathrm{X}$ ' that is to be classified. If ' $\mathrm{X}$ ' doesn't belong to any of the class belonging to model, say, ' $\mathrm{Mi}$ ', then ' $\mathrm{X}$ ' is an outlier with respect to model ' $\mathrm{Mi}$ ' belonging to the ensemble.

Definition 4: F-Outlier: Assume ' $\mathrm{X}$ ' is an instance that is to be classified. If ' $\mathrm{X}$ ' is an outlier with respect to each and every model ' $\mathrm{Mi}$ ' belonging to ensemble ' $\mathrm{L}$ ', then ' $\mathrm{X}$ ' is said to be 'F-Outlier'. That is ' $\mathrm{X}$ ' is an outlier with respect to the complete ensemble of model ' $\mathrm{L}$ '

\section{A. Training Phase}

This is the first step towards building the classification technique. In our technique, we use a fixed size data chunk. We divide the data into fixed size chunks. Each chunk contains 2000 instances in it. Totally we form three

Also many of the strategies developed to detect novel chunks of equal size. After that we apply the k-Medoid classes are of two main types: parametric and non- clustering technique to these chunks individually. By parametric. Parametric approaches assume that data applying clustering to one data chunk we generate one follows certain distribution and then by using the classification model ' $\mathrm{Mi}$ ' of our ensemble. In this we parameters of the normal data it calculates the parameters generate an ensemble containing three classification of the distribution. If an instance is found that doesn't models. We apply k-Medoid clustering technique in our follow the set distribution parameters, it is declared as an system because it works with data that contains outliers. instance belonging to novel class. Hence such strategies Models are built from the recent data chunk. We then store are restricted to certain data distributions. Non-parametric the summaries of the clusters obtained. In this we store the techniques never make any assumptions about data number of clusters belonging to each model 'Mi' and the distribution and hence are never restricted by data set of words ' $\mathrm{Si}$ ' defining each cluster or belonging to each distribution. The technique that we have developed is also cluster.

non-parametric. Also most of the techniques developed for 
Ensemble of model classifies the test instance as follows: first retrieve its set of words ' $S_{k}$ ', then we compare ' $S_{k}$ ' Assume ' $\mathrm{X}$ ' to a test instance that is to be classified. First with the set of words of various clusters belonging to of all it will be sent to each and every model 'Mi' of the model 'Mi' by performing the operation of set intersection ensemble ' $L$ '. It will be compared to each cluster on $S_{k}$ and set of words ' $S_{j}$ ' of various classes ' $C_{j}$ '. We belonging to each model of the ensemble. If ' $\mathrm{X}$ ' will be then compare the obtained result against the set of words found belonging to an existing class ' $\mathrm{C}$ ' then it will ' $\mathrm{S}_{\mathrm{k}}$ ' of instance. If more than $50 \%$ of words instance are classified by the model containing the class ' $\mathrm{C}$ '. But if it present in the cluster word set ' $\mathrm{S}_{\mathrm{i}}$ ' then we deduce that the is detected as an outlier (OUT) by all of the models outlier is present due to concept-drift in the data. We store contained in the ensemble then it will be classified as final such outliers in the array named CONDRIFT. outlier (F-OUT).

\section{2) Handling Concept-Drift:}

\section{B. Outlier Detection}

After detecting concept-drift, our next task to handle it properly. Here we obtain the set of words ' $\mathrm{S}_{\mathrm{k}}$ ' of each After generating the three classification models we take instance in CONDRIFT. We then perform the operation of next step to detect the outliers. For getting the outliers of set difference on the word set ' $S_{k}$ ' and the set of words ' $S_{i}$ ' the ensemble we make use of the stored summaries of the of the class ' $\mathrm{C}_{\mathrm{i}}$ ' from which the instance has drifted. The clusters that were in the form of number of clusters and the resultant obtained contains the new features that have set of words ' $\mathrm{Si}$ ' of the clusters. Assume if ' $\mathrm{X}$ ' is an arrived recently in the class or the drifting features. These instance that is to be classified or is to be detected as an new features are stored in a vector named DRIFTWORD outlier.

We first obtain the set of words $S(X)$ of the instance ' $X$ ' along the information about the original class ' $\mathrm{C}_{\mathrm{i}}$ '. Then and compare it with the set of words ' $\mathrm{Si}$ ' of the class ' $\mathrm{C} i$ ' obtain the unique drift words. In the next step we construct belonging to model ' $\mathrm{Mj}$ '. If the set $\mathrm{S}(\mathrm{X})$ matches with the a matrix CHKMAT. Initially the values in the matrix are set of words ' $\mathrm{Si}$ ' of any of the class ' $\mathrm{Ci}$ ' belonging to odel set to one. The rows of the matrix CHKMAT denote the ' $\mathrm{Mj}$ ', then it is classified accordingly. Otherwise it is unique drift words and the columns of the matrix denote detected as an outlier to the model ' $\mathrm{Mj}$ '. In this way we the probable classes ' $\mathrm{C}_{\mathrm{j}}$ ' of the model 'Mj'. For each obtain the outliers $\left(\mathrm{OUT}_{\mathrm{j}}\right)$ with respect to each and every occurrence of the unique drift word ' $\mathrm{W}_{\mathrm{m}}$ ' belonging to model.

class ' $\mathrm{C}_{\mathrm{j}}$ ', we increment the matrix value CHKMAT $[\mathrm{m}, \mathrm{j}]$ In the second step we try to obtain the final outliers of the by 1 . We then set a threshold value. If the value CHKMAT ensemble of models. Here we generate an array of outliers [m, j] exceeds the threshold value then the unique drift $\left(\mathrm{OUT}_{\mathrm{j}}\right)$ with respect to every model ' $\mathrm{Mj}$ '. We then see word ' $\mathrm{Wm}$ ' is appended to the feature set of the class ' $\mathrm{C}_{\mathrm{j}}$ '. whether an instance present in one outlier vector is also In this way the new drifting features are added to the contained in the other two outlier vectors also. If so then it original model.

is declared as final outlier with respect to the ensemble

' $L$ '. we store all such final outliers in vector 'FOUT'.

Algorithm1. F_OUT

Input: Models $\mathrm{Mi}$ and instances 'X'.

Output: FOUT (Vector containing outliers of the model).

1: For each model 'Mi' in $\mathrm{M}$

2: $\quad$ If $S(X) \in C j^{\prime} M i$

3: Append name of class ' $C_{j}$ ' to test instance ' $X$ '.

4: $\quad$ else

5: $\quad$ Add ' $\mathrm{X}$ ' to $\mathrm{OUT}_{\mathrm{i}}$ vector.

6: $\quad$ End if.

7: End for.

D. Concept-Evolution

1) Detection of concept-evolution :

Here we again take into consideration the FOUT vector. If the instance $\mathrm{OUT}_{\mathrm{k}}$ belonging to the FOUT vector does not qualify the above criteria set for concept-drift, i.e. If the instance does not belong to any of the class ' $\mathrm{C}_{\mathrm{j}}$ ' belonging to any of the model ' $\mathrm{Mj}$ ' of the ensemble, then we deduce that the reason its occurrence is concept-evolution. We store these instances in the vector CONEVOLUTION. The main criteria that needs to be fulfilled is that more than $50 \%$ words of the instance in the FOUT must not match 8: FOUT $=$ Intersection operation $\left(\mathrm{OUT}_{1}, \mathrm{OUT}_{2} \ldots, \mathrm{OUT}_{\mathrm{i}}\right)$ with the set of words of any of the class.

9: End algorithm.

2) Handling Concept-Evolution:

When we try to handle concept-evolution we not only need to find the novel class. We also need to detect the occurrence of multiple novel classes. We make use of

\section{1) Detection of Concept-Drift :} Once we obtain the FOUT vector, we must know that CONEVOLUTION vector. We know that this vector
outliers present in it are generated due to three main contains the instances belonging to one or more novel reasons. These three reasons are outliers concept-drift, classes. We simply apply the k-Medoid clustering concept-evolution and noise. Our next step is to separate technique to the CONEVOLUTION vector. By doing so, the outliers based on the cause of their occurrence. First of we obtain the different novel classes present in the vector. all we try to obtain the outliers due to concept-drift and try At the end we simply add the summaries of the data to one to handle them. We try to detect it in the following of the classification models that were generated initially. manner. For an instance $\mathrm{OUT}_{\mathrm{k}}$ belonging to FOUT, we 
In this way the ensemble is updated. We try to present the algorithms in the next full implementation paper.

\section{DATA SETS}

Our algorithm requires that the data is not multi-valued and multi-labeled. The data instance must belong to one class only.

\section{A. 4 University Data Set:}

Initially we began our work with the above data set. It was present in the form of HTML pages. We applied preprocessing to it and obtained the data in the tabular form. After applying preprocessing we found that the data obtained was multi-valued and multi-labeled. This was against our assumption. Hence we could not continue with this data set.

\section{B. NASA Aviation Safety Reporting System.}

NASA ASRS dataset maintains a record of various accidents taking place in the air industry. This data set can be downloaded from the official website of NASA. The data was already present in the tabular form. With each accident there is an anomaly associated with it. Like aircraft problem: critical, aircraft problem: less severe etc. this event anomaly can be considered as a class. But our data was multi-valued and multi-labeled also. So first we made attempts to remove multi-valued and multi-labeled data to meet the requirement of our algorithm. We then found that there were instances that had incomplete information. We also removed such data from the data set to make it more proper and informative. Our dataset finally contained six existing classes and two novel classes. It contained concept-drift and concept-evolution also.

\section{CONCLUSION AND FUTURE SCOPE}

We in our approach are presenting a string based approach to classify the data streams. We are able to handle conceptdrift, infinite-length and concept-evolution. We are not able to handle feature-evolution that efficiently. We are also able to detect multiple novel classes present in the data simultaneously. Other researchers have used the strategy based on distances. These are quite different from our strategy. We will be presenting our algorithms along with the results once they are tested and developed efficiently. in future we can also work to make the chunk size variable. We can also work to handle featureevolution effectively.

\section{REFERENCES}

[1] C.C. Aggarwal, J. Han, J. Wang, and P.S. Yu, "A Framework for On-Demand Classification of Evolving Data Streams," IEEE Trans. Knowledge and Data Eng., vol. 18, no. 5, pp. 577-589, May 2006.

[2] M.M. Masud, J. Gao, L. Khan, J. Han, and B.M. Thuraisingham, "Classification and Novel Class Detection in Data Streams with Active Mining,".

[3] Y. Yang, X. Wu, and X. Zhu, "Combining Proactive and Reactive Predictions for Data Streams," Proc. ACM SIGKDD 11th Int'l Conf. Knowledge Discovery in Data Mining, pp. 710-715, 2005.

[4] E. J. Spinosa, A. P. de Leon F. de Carvalho, and J. Gama. Clusterbased novel concept detection in data streams applied to intrusion detection in computer networks. In ACM SAC, pages 976-980, 2008.
[5] OLINDDA: A Cluster Based Approach for Detecting Novelty and Concept-Drift in Data Stream by Eduardo J. Spinosa, Andr'e Ponce de Leon F.de Carvalho, Jo ao Gama in ACM Symposium of Applied Computing $S A C^{\prime} 07$

[6] B. Wenerstrom and C. Giraud-Carrier, "Temporal Data Mining in Dynamic Feature Spaces," Proc. Sixth Int'l Conf. Data Mining (ICDM), pp. 1141-1145, 2006

[7] M.M. Masud, J. Gao, L. Khan, J. Han, and B.M. Thuraisingham, "Classification and Novel Class Detection in Concept-Drifting Data Streams under Time Constraints," IEEE Trans. Knowledge and Data Eng,vol. 23, no. 6, pp. 859-874, June 2011.

[8] M.M. Masud, J. Gao, L. Khan, J. Han, and B.M. Thuraisingham, "Classification and Novel Class Detection in Feature Based Stream Data," IEEE Trans. Knowledge and Data Eng, vol. 25, no. 7, July 2013.

[9] M.M. Masud, J. Gao, L. Khan, J. Han, and B.M. Thuraisingham, "Integrating Novel Class Detection with Classification for ConceptDrifting Data Streams, ” IEEE Trans. Knowledge and Data Eng , vol. 25, no. 7, July 2009.

[10] M.M. Masud, Q. Chen, J. Gao, L. Khan, J. Han, and B.M. Thuraisingham, "Classification and Novel Class Detection of Data Streams in a Dynamic Feature Space," Proc. European Conf. Machine Learning and Knowledge Discovery in Databases (ECMLPKDD), pp. 337-352, 2010.

[11] M.M. Masud, Q. Chen, L. Khan, C. Aggarwal, J. Gao, J. Han, and B.M. Thuraisingham, "Addressing Concept-Evolution in ConceptDrifting Data Streams," Proc. IEEE Int'l Conf. Data Mining (ICDM), pp. 929-934, 2010.

[12] A Review of Method of Stream data classification through Optimized Feature Evolution Process by Archana Bopche, Malti Nagle and Hitesh Gupta International Journal Of Engineering And Computer Science ISSN:2319-7242Volume 3 Issue 1 January, 2014 Page No.3778-3783. 